Article

\title{
Design, Synthesis and Anti-HIV Integrase Evaluation of 4-Oxo- 4H-quinolizine-3-carboxylic Acid Derivatives
}

\author{
Yi-Sheng Xu ${ }^{1,2}$, Cheng-Chu Zeng ${ }^{1, *}$, Zi-Guo Jiao ${ }^{1}$, Li-Ming Hu ${ }^{1}$ and Ru-gang Zhong ${ }^{1}$
}

1 College of Life Science and Bio-Engineering, Beijing University of Technology, Beijing 100124, P. R. China

2 Atmospheric Chemistry \& Aerosol Research Department, Chinese Research Academy of Environmental Sciences, Beijing 100012, P. R. China; E-mails: xuys@craes.org.cn (Y-S.X)

* Author to whom correspondence should be addressed; E-Mail: zengcc@bjut.edu.cn; Tel.: +86-1067396211; Fax: +86-10-67392001.

Received: 30 December 2008; in revised form: 16 January 2009 / Accepted: 6 February 2009 / Published: 19 January 2009

\begin{abstract}
Oxo-4H-quinolizine-3-carboxylic acid derivatives bearing sulfamido, carboxylamido, benzimidazole and benzothiazole substituents have been designed and synthesized. The structures of these new compounds were confirmed by ${ }^{1} \mathrm{H}-\mathrm{NMR},{ }^{13} \mathrm{C}-$ NMR, IR and ESI (or HRMS) spectra. Compounds were screened for possible HIV integrase inhibitory activity.
\end{abstract}

Keywords: 4-Oxo-4H-quinolizine-3-carboxylic acid derivatives; HIV-1 integrase; $\mathrm{Mg}^{2+}$ binding; Aryl diketo acids.

\section{Introduction}

Human immunodeficiency virus type 1 (HIV-1) is the major pathogen of the infection of acquired immunodeficiency syndrome (AIDS) [1]. In the past two and half decades, various compounds have been developed as medicinal candidates for the treatment of HIV infections aiming at one or several steps of the HIV-1 life cycle [2-5] such as absorption, entry, fusion, un-coating, reverse transcription, integration, transcription and maturation. Owing to the facts that HIV integrase (IN) is the indispensable enzyme in the replication of the life cycle for the HIV virus and no cellular homologues 
are found in humans [6-9], inhibitors targeted selectively at HIV IN are expected to have low cytotoxicity and thus development of HIV IN inhibitors has attracted more and more attention.

To this end, aryl diketoacids compounds have been reported to be the most promising HIV IN inhibitors [10-12]. Structurally, this type of HIV IN inhibitors consists of two main domains, namely, a diketoacid subunit and an aromatic moiety with one or two arylalkyl substituents. The former serves as the pharmaphore and is proposed to sequester the divalent cofactors $\left(\mathrm{Mg}^{2+}\right)$ in the IN active site and thus block access of a host DNA to the integrase [13,14]. Meanwhile, the aromatic moiety as hydrophobic domain, typically an indole or benzene moiety, is responsible for the selectivity to the strand transfer (ST) reaction $[15,16]$.

As part of our continuing efforts directed towards the development of potential HIV-1 integrase inhibitors, we have investigated the chemical and electrochemical synthesis of styrylquinolines [17], quinoxalones [18,19] and polyhydroxylated aromatics [20,21]. Based on the structural characteristics of diketo acid-type compounds and their molecular mechanism as HIV IN inhibitors, very recently, a series of 4-oxo-1,4-dihydro-1,5-naphthyridine-3-carboxylic acids have been designed and synthesized by our group (see I, Figure 1) [22]. In view of the fact that the 4-oxo-quinolizine-3-carboxylic acid moiety displays high selectivity and binding ability to $\mathrm{Mg}(\mathrm{II})$ (KMG-103 and KMG-104, Figure 1) $[23,24]$ and other derivatives show antibacterial and antimicrobial activities [25-27], in the present work, we further modified the main core via addition of a bulky group, such as an aryl sulfonamido, aryl carboxylamido, benzimidazole or benzothiazole chosen as the side hydrophobic chain and placed at the C-1 position of the 4-oxo-quinolizine-3-carboxylic acid scaffold. Thus, a series of 4-oxoquinolizine-3-carboxylic acid derivatives 8, 9, 12 and 14 were synthesized. Results show that, although these compounds could bind $\mathrm{Mg}^{2+}$ in $\mathrm{CH}_{3} \mathrm{CN}$ solution, no obvious anti-HIV IN activity is observed.

Figure 1. The design of 4-oxo-quinolizine-3-carboxylic acid derivatives as potential HIV IN inhibitors.

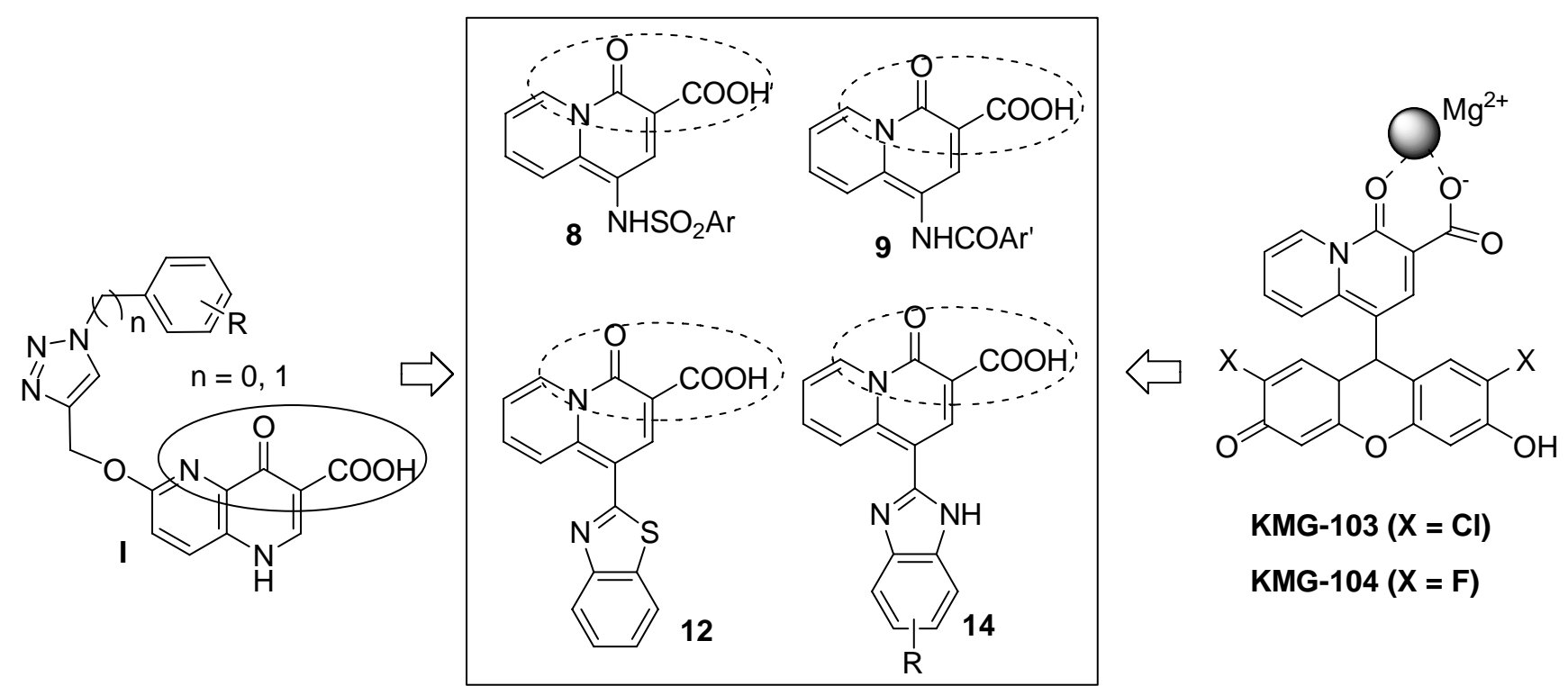




\section{Results and Discussion}

Synthesis of 4-oxo-4H-quinolizine-3-carboxylic acid derivatives 8, 9, 12 and 14

The synthesis of 4-oxo-4H-quinolizine-3-carboxylic acid derivatives 8 and $\mathbf{9}$ is described in Scheme 1. Ethyl 4-oxo-4H-quinolizine-3-carboxylate (3) was synthesized in good yield starting from 2-methylpyridine according to a literature procedure [24]. Nitration of $\mathbf{3}$ afforded compound $\mathbf{4}$, which was reduced by $\mathrm{Na}_{2} \mathrm{~S}_{2} \mathrm{O}_{4}$ to generate the key intermediate ethyl 1-amino-4-oxo-4H-quinolizine-3carboxylate (5). In the presence of pyridine, compound 5 was treated with sulfonyl chloride or acyl chloride to give compounds 6 and 7, respectively. The target 4-oxo-4H-quinolizine-3-carboxylic acids 8 and 9 were finally obtained after hydrolysis in $\mathrm{NaOH} /$ methanol followed by neutralization. During the nitration procedure, the speed of addition of nitric acid should be carefully controlled, otherwise, the reaction mixture would become red and the amount of by-products increased. In addition, $\mathrm{Na}_{2} \mathrm{~S}_{2} \mathrm{O}_{4}$ was used to reduce nitro group and generated key intermediate 5 , due to the benign reaction conditions (usually at room temperature using water as solvent) and simple workup.

Scheme 1. The synthesis of 4-oxo-4H-quinolizine-3-carboxylic acid derivatives 8 and 9.

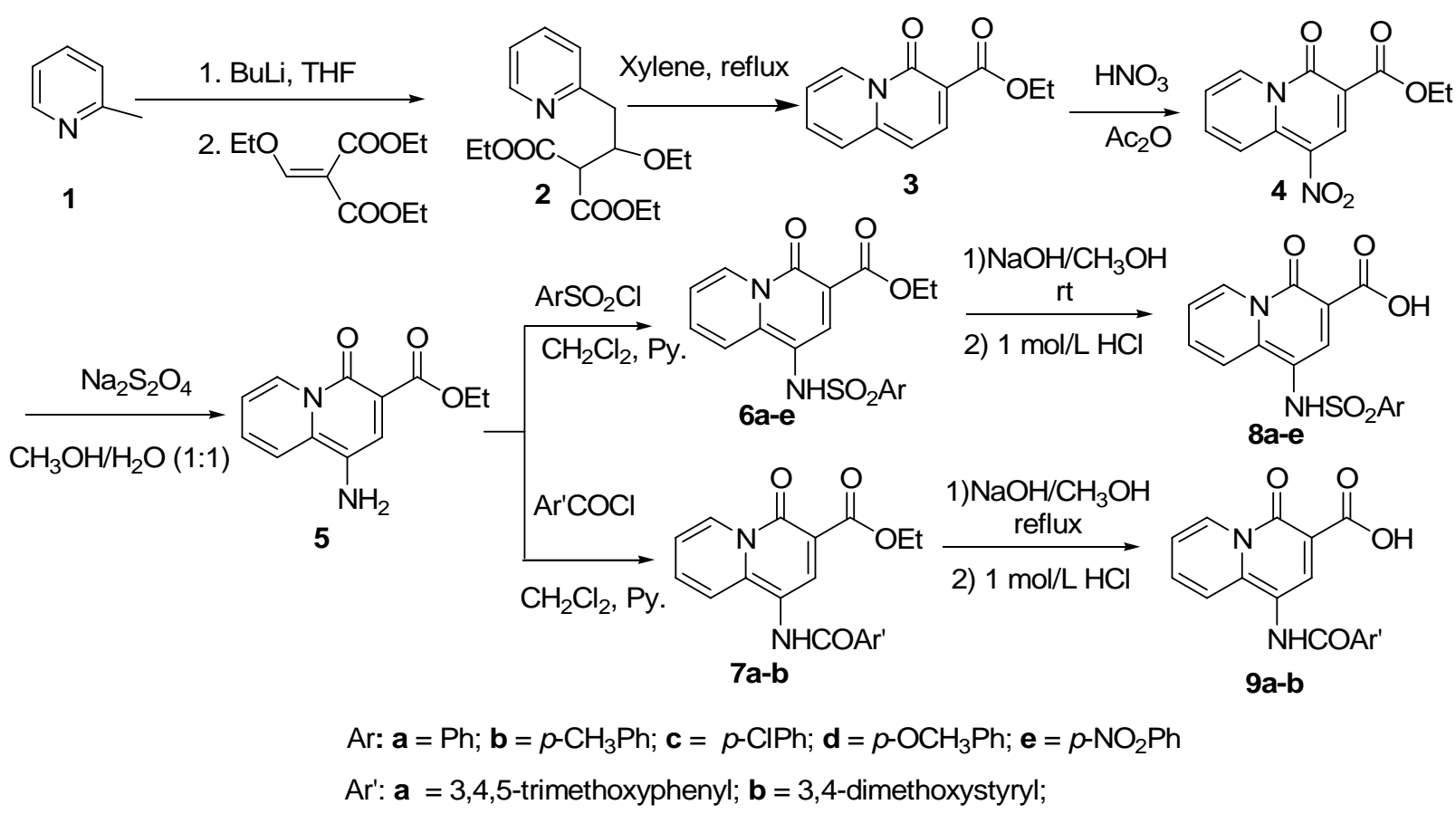

With 3 in hand, we then synthesized benzothiazole- and benzimidazole-substituted 4-oxo- $4 \mathrm{H}$ quinolizine-3-carboxylic acid derivatives 12 and 14. As shown in Scheme 2, reaction of compound 3 with DMF and $\mathrm{POCl}_{3}$ generated intermediate 10 [24], which reacted with 2-aminothiophenol [28, 29] followed by subsequent hydrolysis to generate 1-(benzothiazole-2-yl)-4-oxo-4H-quinolizine-3carboxylic acid (12). Similarly, compound 10 underwent oxidative condensation with $o$-phenylenediamine derivatives in nitrobenzene [30] yielded compounds 13a-c, which were hydrolyzed [31] to obtain 1-(benzimidazole-2-yl)-4-oxo-4H-quinolizine-3-carboxylic acids 14a-c. It should be pointed out 
that two benzimidazole isomers were formed for each of 13b-c and 14b-c, that could not be separated each other through conventional procedures.

It is noteworthy that the conditions for the hydrolysis of $\mathbf{1 1}$ and $\mathbf{1 3}$ were not similar to those used for the synthesis of $\mathbf{8}$ and $\mathbf{9}$. The reaction proceeded very slowly in the $\mathrm{NaOH} / \mathrm{MeOH}$ system. For example, after 48 hours stirring at room temperature, the reaction mixtures were still slurries and most of the starting materials were present in the reaction system. Consequently, $\mathrm{KOH}$ in $80 \%$ aqueous ethanol was used for the synthesis of $\mathbf{1 2}$ and 14. As the reaction proceeded the slurry of the reaction mixture turned clear and the products precipitated when $1 \mathrm{~mol} \cdot \mathrm{L}^{-1}$ aqueous $\mathrm{HCl}$ was added.

Scheme 2 Synthesis of 4-oxo-4H-quinolizine-3-carboxylic acid derivatives 12 and 14.

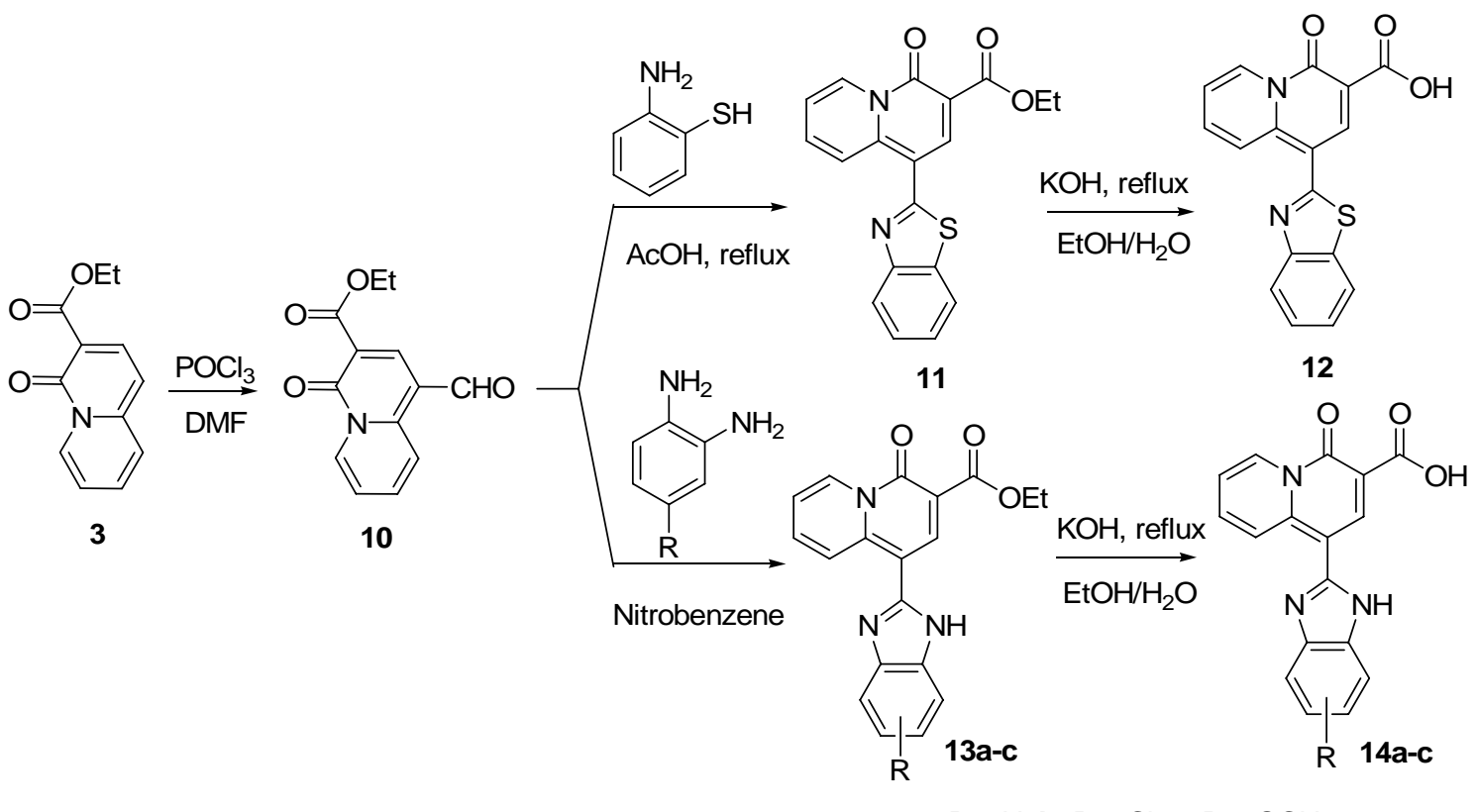

a, $\mathrm{R}=\mathrm{H} ; \mathbf{b}, \mathrm{R}=\mathrm{Cl} ; \mathbf{c}, \mathrm{R}=\mathrm{OCH}_{3}$

Structural analysis

The structures of all new 4-oxo-4H-quinolizine-3-carboxylic acid derivatives 6-14 were confirmed by ${ }^{1} \mathrm{H}-\mathrm{NMR},{ }^{13} \mathrm{C}-\mathrm{NMR}$, IR and ESI-MS (or HRMS) spectra. In the ${ }^{1} \mathrm{H}-\mathrm{NMR}$ spectra of ethyl 4-oxo$4 H$-quinolizine-3-carboxylates 6, 7, 11, 13, the 4-oxo- $4 H$-quinolizine ring proton signals appeared at lower field compared with those of the benzene ring. For example, in the ${ }^{1} \mathrm{H}-\mathrm{NMR}$ spectra of $\mathbf{6 b}$, the two doublets at $9.43 \mathrm{ppm}$ and $8.28 \mathrm{ppm}$ were ascribed to the protons at C-5 and C-8 of the 4-oxo- $4 \mathrm{H}$ quinolizine ring, respectively, while the protons at C-6 and C-7 exhibited two double doublets at 7.76 ppm and $7.29 \mathrm{ppm}$. The proton at $\mathrm{C}-2$ resided at $7.63 \mathrm{ppm}$. A high field AB system (7.31 ppm and 7.64 ppm) corresponded to the signals of the four benzene ring protons.

In the ${ }^{1} \mathrm{H}-\mathrm{NMR}$ of target compounds 8, 9, 12, 14a, one sharp singlet in the $\delta$ 13.7-14.1 range was observed, which disappeared after the $\mathrm{D}_{2} \mathrm{O}$ exchange and was consequently attributed to the $\mathrm{COOH}$ proton. For example, the proton signals of the $\mathrm{COOH}$ groups of compounds 8a and 14a were located at $13.7 \mathrm{ppm}$ and $13.8 \mathrm{ppm}$, respectively. In addition, compound 14a has a strong singlet at $\delta 13.7 \mathrm{ppm}$, which demonstrated the existence of a $\mathrm{N}-\mathrm{H}$ proton in the benzimidazole ring. In the ${ }^{13} \mathrm{C}-\mathrm{NMR}$ spectra of these compounds one $\mathrm{C}=\mathrm{O}$ peak at about $166 \mathrm{ppm}$ and one around $159 \mathrm{ppm}$ corresponded to the signals of the 3-carboxylic acid and carbonyl groups in the quinolizine rings. The IR spectra of these 
compounds further support their postulated structures. There were several medium broad bands at $3,200 \sim 3,400 \mathrm{~cm}^{-1}$ corresponding to $\mathrm{O}-\mathrm{H}$ and N-H bond and strong bands at 1,690 1,730 $\mathrm{cm}^{-1}$ due to $\mathrm{C}=\mathrm{O}$ bonds. The mass spectra of compounds $\mathbf{8 c}, \mathbf{1 3 b}$ and $\mathbf{1 4 b}$ exhibited a $\mathrm{M}+2$ signal, whose intensity was $1 / 3$ of the molecular ion signal, and corresponding to the $\mathrm{Cl}$ isotopes in these compounds.

\section{Preliminary investigation of $\mathrm{Mg}^{2+}$ binding}

To elucidate a two-metal inhibition model, very recently, Kawasuji et al [32] performed metal titration studies of 2-hydroxy-3-heteroarylacrylic acid derivatives and observed a two-step shift of the UV-Vis spectra upon the addition of $\mathrm{Mg}^{2+}$ if these compounds exhibited HIV-IN inhibitory activity. On the contrary, simple change of UV-Vis absorption curves or no shift was found for their mutated compounds which do not inhibit HIV integrase. Inspirited by this assumption, preliminary ion binding experiments were also carried out by UV-Vis spectroscopy, using compound 14a as an example. Due to the low solubility of 14a in water, $\mathrm{CH}_{3} \mathrm{CN}$ was used to prepare the stock solution for the UV-Vis measurements. As shown in Figure 2, for the free compound 14a, two intense absorption bands at 352 $\mathrm{nm}$ and $414 \mathrm{~nm}$ were observed in the $320-550 \mathrm{~nm}$ range. As the $\mathrm{Mg}^{2+}$ concentration (as perchlorate salt) increased, the absorption intensity of the peak at $414 \mathrm{~nm}$ first decreased and then the wavelength shifted blue to around $402 \mathrm{~nm}$. Figure 3 further demonstrated the selectivity of compound $14 \mathbf{a}^{2} \mathrm{Mg}^{2+}$. In the presence of a 100-fold equivalent amount of $\mathrm{Na}^{+}$or $\mathrm{K}^{+}$, no obvious change was observed. However, the $\mathrm{Mg}^{2+}$ induced a blue shift of the absorption curve of compound 14a. Similar behaviors were also observed for other divalent metals, including $\mathrm{Mn}^{2+}, \mathrm{Co}^{2+}, \mathrm{Cu}^{2+}, \mathrm{Ca}^{2+}$ and $\mathrm{Ni}^{2+}$ (not shown in Figure 3).

Figure 2. Changes of UV-vis spectra with $14 a$ in $\mathrm{CH}_{3} \mathrm{CN}$ on addition of $0-16 \times 10^{-6} \mathrm{~mol} \cdot \mathrm{L}^{-1}$ of $\left[\mathrm{Mg}^{2+}\right]$ at $25^{\circ} \mathrm{C}$.

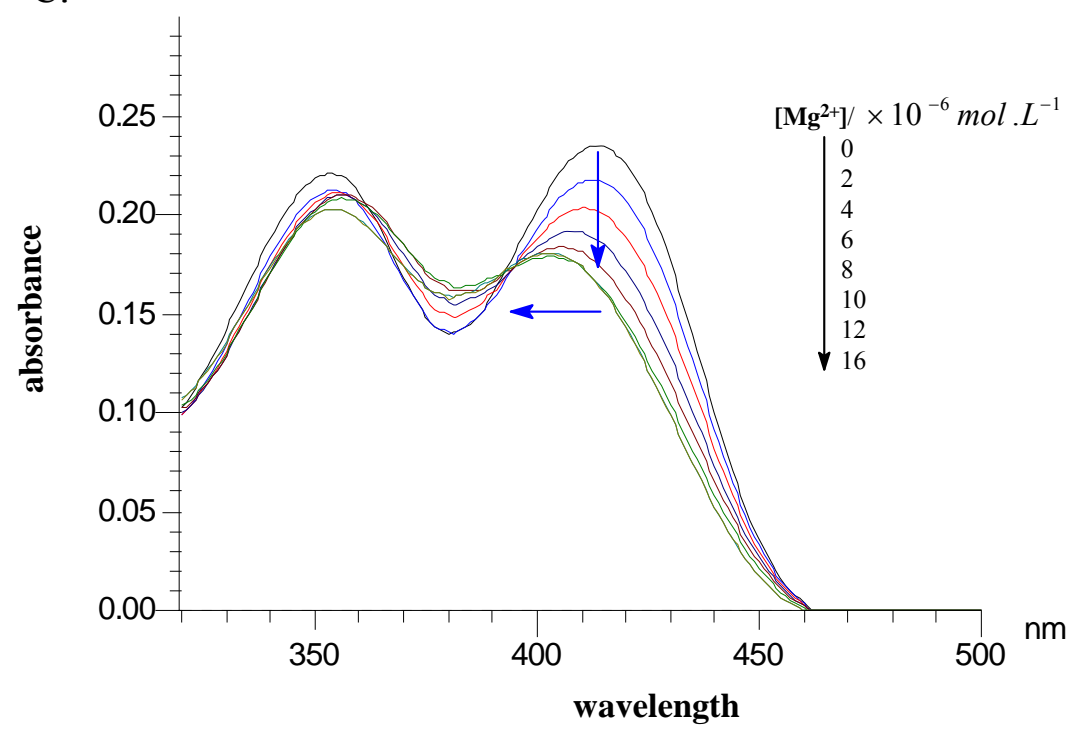

Conditions: $\left[\mathrm{Mg}^{2+}\right]=2 \times 10^{-3} \mathrm{M} .[\mathbf{1 4 a}]=2 \times 10^{-5} \mathrm{MBlack}: 0 \times 10^{-6} \mathrm{~mol} \cdot \mathrm{L}^{-1}$, blue: $2 \times 10^{-6} \mathrm{~mol} \cdot \mathrm{L}^{-1}$, red: $4 \times 10^{-6} \mathrm{~mol} \cdot \mathrm{L}^{-1}$, navy: $6 \times 10^{-6} \mathrm{~mol} \cdot \mathrm{L}^{-1}$, brown: $8 \times 10^{-6} \mathrm{~mol} \cdot \mathrm{L}^{-1}$, green: $10 \times 10^{-6} \mathrm{~mol} \cdot \mathrm{L}^{-1}$, dark cyan: $12 \times 10^{-6} \mathrm{~mol} \cdot \mathrm{L}^{-1}$, brass: $16 \times 10^{-6} \mathrm{~mol} \cdot \mathrm{L}^{-1}$. 
The titration experiment of $\mathbf{1 4 a}$ with $\mathrm{Mg}^{2+}$ further demonstrated that the binding ratio is $1: 1$, which is consistent with that of KMG-103 and KMG-104 to $\mathrm{Mg}^{2+}$, indicating that the introduction of benzimidazole substituent on the $\mathrm{C}-1$ position of the 4-oxo- $4 H$-quinolizine-3-carboxylic acid scaffold does not affect its $\mathrm{Mg}^{2+}$ binding ability. In a word, a two-step shift of the absorption curve of compound 14a upon the addition of $\mathrm{Mg}^{2+}$ was observed, similar to 2-hydroxy-3-heteroaryl acrylic acid derivatives reported by Kawasuji et al [32]. These UV-Vis results indicated that our compounds might interact with metal ions $\left(\mathrm{Mg}^{2+}\right)$ in the active site of HIV IN and thus offer a piece of in vitro indication of possible HIV IN inhibitory activity of these new compounds, in spite of no strict demonstration of correction between the inhibitory activity and the metal affinity.

Figure 3. UV-Vis absorption spectra of free 14a and 14a in the presence of $\mathrm{K}^{+}, \mathrm{Na}^{+}$or $\mathrm{Mg}^{2+}$ ion in $\mathrm{CH}_{3} \mathrm{CN}$ solution.

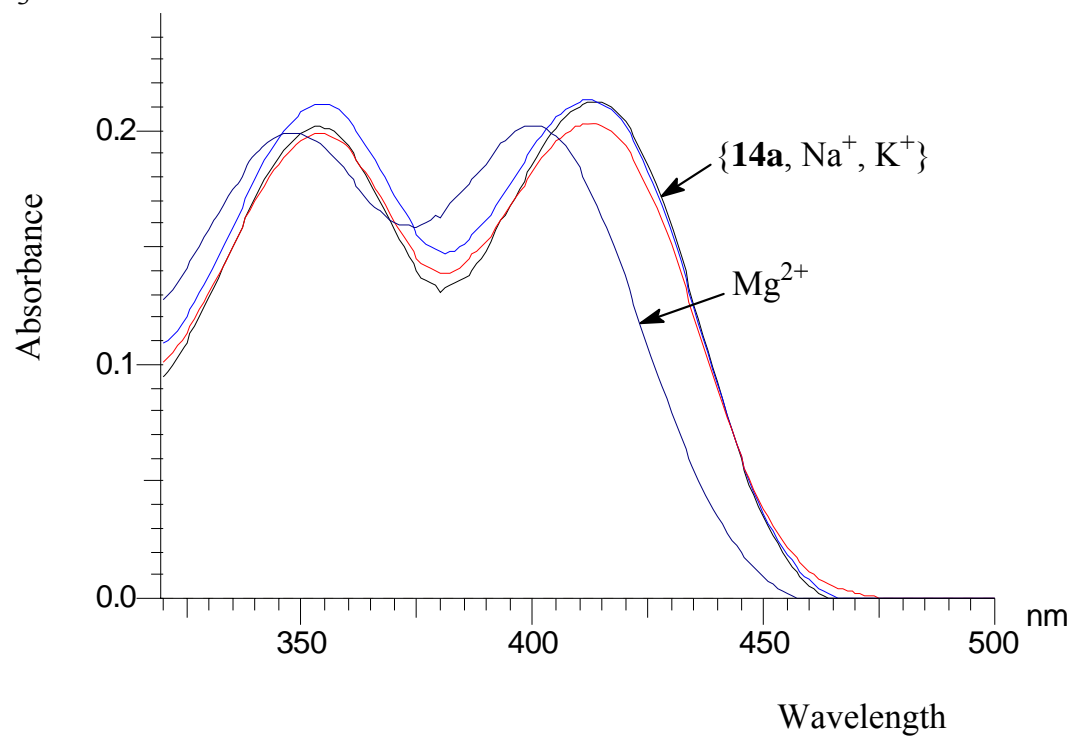

Conditions: $[\mathbf{1 4 a}]=2 \times 10^{-5} \mathrm{M} ;\left[\mathrm{Mg}^{2+}\right]=\left[\mathrm{K}^{+}\right]=\left[\mathrm{Na}^{+}\right]=2 \times 10^{-3} \mathrm{M}$; Black: 14a, blue: $\mathrm{Na}^{+}$complex, red: $\mathrm{K}^{+}$complex, navy: $\mathrm{Mg}^{2+}$ complex.

HIV integrase inhibitory activities of 4-oxo-4H-quinolizine-3-carboxylic acid derivatives

The target compounds $\mathbf{8}, \mathbf{9}, \mathbf{1 2}, \mathbf{1 4}$ were screened against purified HIV IN to determine any potential inhibitory activity. Thus, a synthesized 30 oligonucleotide and a 20 oligonucleotide were used as donor DNA and target DNA, respectively, which were purchased from Shanghai Sangon Biological Engineering Technology \& Services Co. Ltd. The donor DNA was biotinylated at the 5' end (5' bio-DNA) and immobilized on streptavidin-coated 96-well microtiter plates. To the donor DNA streptavidin-coated 96-well microtiter plate were added test sample, 3' digoxygenin-labeled target DNA (3' dig-DNA) and recombinant HIV integrase and subjected to incubation in optimized condition.

The plate was further washed with phosphate-buffered saline (PBS) containing $0.1 \%$ Tween ${ }^{\circledR}-20$ and anti-digoxigenin-peroxidase (POD, $100 \mu \mathrm{L}$ ) was added to each well. After incubation at $37^{\circ} \mathrm{C}$ for $60 \mathrm{~min}$, the plate was washed with PBS containing $0.1 \%$ Tween ${ }^{\circledR}-20$. The digoxygenin-labeled products were visualized by adding a POD substrate, tetramethylbenzidine (TMB). The colorimetric 
reaction was stopped by addition of $0.5 \mathrm{~mol} / \mathrm{L} \mathrm{H}_{2} \mathrm{SO}_{4}(100 \mu \mathrm{L})$, and the absorbance was measured at $450 \mathrm{~nm}$ on a microplate reader. The total inhibitory effect (including inhibiting $3^{\prime}$ process reaction and strand transfer reaction) were obtained by comparing the absorbance of drug group with control group (without test compound). As shown in Table 1, the values of $\mathrm{IC}_{50}$ of these compounds are more than $100 \mu \mathrm{g} / \mathrm{mL}$, which mean a low inhibitory activity to HIV integrase.

The low HIV IN inhibitory activity of these designed 4-oxo-4H-quinolizine-3-carboxylic acid derivatives might stem from various cofactors. Structurally, compounds 8, 9, 12 and 14 possess a chelating scaffold suitable for binding only one ion. However, a two-metal chelating scaffold to form respectively a five-membered and a six-membered system is required according to the two-metal coordinating model for diketoacid-type HIV IN inhibitors. Therefore, the selective binding to $\mathrm{Mg}^{2+}$ is not enough, the two-metal binding scaffold is also necessary in the design of diketoacid-type HIV IN inhibitors. In addition, an appropriate substituent at appropriate position of the chelating scaffold is also essential to ensure potential inhibitory activity.

Table 1. Integrase inhibitory activity data of 4-oxo-4H-quinolizine-3-carboxylic acid derivatives.

\begin{tabular}{llllll}
\hline Sample & $\begin{array}{l}\text { Initial concentration } \\
(\mu \mathrm{g} / \mathrm{mL})\end{array}$ & $\begin{array}{l}\mathrm{IC}_{50} \\
(\mu \mathrm{g} / \mathrm{mL})\end{array}$ & Sample & $\begin{array}{l}\text { Initial concentration } \\
(\mu \mathrm{g} / \mathrm{mL})\end{array}$ & $\begin{array}{l}\mathrm{IC}_{50} \\
(\mu \mathrm{g} / \mathrm{mL})\end{array}$ \\
\hline $\mathrm{S}-\mathrm{y}^{1}$ & 12 & 0.56 & $\mathbf{9 a}$ & 100 & $-{ }^{2}$ \\
$\mathbf{8 a}$ & 100 & -2 & $\mathbf{9 b}$ & 100 & $-{ }^{2}$ \\
$\mathbf{8 b}$ & 100 & -2 & $\mathbf{1 2}$ & 100 & $-{ }^{2}$ \\
$\mathbf{8 c}$ & 100 & -2 & $\mathbf{1 4 a}$ & 100 & $-{ }^{2}$ \\
$\mathbf{8 d}$ & 100 & -2 & $\mathbf{1 4 b}$ & 100 & $-{ }^{2}$ \\
$\mathbf{8 e}$ & 100 & -2 & $\mathbf{1 4 c}$ & 100 & ${ }^{2}$ \\
\hline
\end{tabular}

${ }^{1}$ S-y provided by the Shanghai Institute of Organic Chemistry was used as positive contrast.

2 "." indicates that the HIV-IN inhibitory effect was less than $50 \%$ at the initial concentration.

\section{Conclusions}

Aryl diketoacid-containing compounds were demonstrated to be the most promising HIV IN inhibitors. Based on the molecular mechanism of aryl diketoacids as HIV IN inhibitors and the selective binding to $\mathrm{Mg}^{2+}$ of 4-oxo- $4 H$-quinolizine-3-carboxylic acid unit, a series of 4-oxo- $4 H_{-}$quinolizine-3-carboxylic acid derivatives, substituted by sulfonoamido, carboxylamido, benzimidazole and benzothiazole groups, were designed and synthesized as diketoacid bioisosters. Their anti-HIV IN activity was evaluated and no obvious inhibitory activities were observed. Based on the present results, in the course of our coming works on development of HIV IN inhibitors, we would have a concept that new types of HIV IN inhibitors would incorporate a two-metal chelating scaffold and a appreciate hydrophobic domain. Moreover, the two-metal chelating scaffold could have an obvious binding affinity to $\mathrm{Mg}^{2+}$. 


\section{Experimental}

\section{General}

All melting points (m.p.) were measured with a XT4A electrothermal apparatus equipped with a microscope and are uncorrected. Infrared spectra (IR) were recorded as thin films on $\mathrm{KBr}$ plates with a Bruker IR spectrophotometer and are expressed in $v\left(\mathrm{~cm}^{-1}\right) .{ }^{1} \mathrm{H}$ - and ${ }^{13} \mathrm{C}-\mathrm{NMR}$ spectra were recorded with an AV $400 \mathrm{M}$ Bruker spectrometer $\left(400 \mathrm{MHz}{ }^{1} \mathrm{H}\right.$ frequency, $100 \mathrm{MHz}{ }^{13} \mathrm{C}$ frequency). Chemical shifts were measured in $\mathrm{CDCl}_{3}$ or DMSO- $d_{6}$ with TMS as internal reference. The MS spectra (ESI) were recorded on a Bruker Esquire 6000 mass spectrometer. HRMS spectra were recorded in the negative ion mode using APEX II FT-ICRMS of Bruker Daltonics Inc. All solvents were of commercial quality and were dried and purified by standard procedures. Compounds 2, 3, 4, 10 were synthesized according to the literature procedure [24].

General procedure for the synthesis of ethyl 1-(substituted phenylsulfonamido)-4-oxo-4H-quinolizine3-carboxylates 6

Compound $4(1.97 \mathrm{~g}, 7.5 \mathrm{mmol})$ was suspended in a 1:1 mixture of methanol and water $(100 \mathrm{~mL})$ in a $250 \mathrm{~mL}$ flask. $\mathrm{Na}_{2} \mathrm{~S}_{2} \mathrm{O}_{4}(7.8 \mathrm{~g}, 45 \mathrm{mmol})$ was then added in one portion at room temperature and the mixture was stirred for about 2 hours. After the reaction, the organic compound was extracted three times using $\mathrm{CH}_{2} \mathrm{Cl}_{2}$ and dried over anhydrous $\mathrm{Na}_{2} \mathrm{SO}_{4}$. After filtration of the $\mathrm{Na}_{2} \mathrm{SO}_{4}$, the solvent was removed under reduced pressure to give $1.0 \mathrm{~g}$ (57\%) of 5 as a red solid, which was used for the following reactions without further purification. The appropriate substituted sulfonyl chloride (3.44 mmol) in $\mathrm{CH}_{2} \mathrm{Cl}_{2}(30 \mathrm{~mL})$ was added dropwise to a $\mathrm{CH}_{2} \mathrm{Cl}_{2}$ solution $(30 \mathrm{~mL})$ containing 5 (3.44 mmol) and dry pyridine $(0.3 \mathrm{~mL})$. The solution changed from red to dark and TLC showed the formation of new compounds with red fluorescence emission. After the reaction, the solution was washed with water and the organic layer was dried over anhydrous $\mathrm{MgSO}_{4}$. After filtration of the $\mathrm{MgSO}_{4}$, the solvent was removed under reduced pressure and the residue was recrystallized by ethyl acetate to give the desired products 6 as yellow solids.

Ethyl 1-phenylsulfonamido-4-oxo-4H-quinolizine-3-carboxylate (6a): Yield 72\%. m.p.: 207-209 ${ }^{\circ} \mathrm{C}$; ${ }^{1} \mathrm{H}-\mathrm{NMR}\left(\mathrm{CDCl}_{3}\right): \delta 1.34\left(\mathrm{t}, 3 \mathrm{H}, J=7.1 \mathrm{~Hz}, \mathrm{CH}_{3}\right), 4.32\left(\mathrm{q}, 2 \mathrm{H}, J=7.1 \mathrm{~Hz}, \mathrm{OCH}_{2}\right), 7.25(\mathrm{dd}, 1 \mathrm{H}, J=$ $7.7 \mathrm{~Hz}, J=5.9 \mathrm{~Hz}), 7.48 \sim 7.91(\mathrm{~m}, 8 \mathrm{H}, \mathrm{Ar}-\mathrm{H}), 9.42(\mathrm{~d}, 1 \mathrm{H}, J=7.1 \mathrm{~Hz},) ;{ }^{13} \mathrm{C}-\mathrm{NMR}\left(\mathrm{CDCl}_{3}\right): \delta 14.4$, 60.9, 105.6, 106.9, 117.3, 122.0, 128.9, 129.3, 130.1, 134.6, 135.5, 138.4, 143.9, 147.2, 154.7, 164.5; IR: v 3491, 3139, 2981, 1734, 1698, $1493 \mathrm{~cm}^{-1}$; MS (ESI): $m / z 370.9$ [M - H] .

Ethyl 1-p-methylphenylsulfonamido-4-oxo-4H-quinolizine-3-carboxylate (6b): Yield 60\%. m.p.: 115$117{ }^{\circ} \mathrm{C} ;{ }^{1} \mathrm{H}-\mathrm{NMR}\left(\mathrm{CDCl}_{3}\right): \delta 1.31\left(\mathrm{t}, 3 \mathrm{H}, J=7.2 \mathrm{~Hz}, \mathrm{CH}_{3}\right), 2.45\left(\mathrm{~s}, 3 \mathrm{H}, \mathrm{PhCH}_{3}\right), 4.30(\mathrm{q}, 2 \mathrm{H}, J=7.2$ $\left.\mathrm{Hz}, \mathrm{CH}_{2}\right), 6.38(\mathrm{~s}, 1 \mathrm{H}, \mathrm{NH}), 7.29 \sim 7.33$ (m, 3H, Ar-H), 7.64(s, 1H, Ar-H), 7.65(d, 2H, J= 7.6 Hz, Ar), $7.77(\mathrm{dd}, 1 \mathrm{H}, J=7.5 \mathrm{~Hz}, \mathrm{~J}=8.0 \mathrm{~Hz}, \mathrm{Ar}-\mathrm{H}), 8.28(\mathrm{~d}, 1 \mathrm{H}, J=8.8 \mathrm{~Hz}, \mathrm{Ar}-\mathrm{H}), 9.43$ (d, 1H, $J=7.1 \mathrm{~Hz})$; ${ }^{13} \mathrm{C}-\mathrm{NMR}\left(\mathrm{CDCl}_{3}\right): \delta 14.6,21.8,61.1,104.9,108.3,117.7,122.9,128.0,130.1,130.1,135.4,135.5$, 
141.5, 144.7, 146.9, 155.1, 165.3; IR: $v$ 3452, 3164, 2972, 1718, 1629, $1490 \mathrm{~cm}^{-1}$; MS (ESI): $\mathrm{m} / z 387.0$ $[\mathrm{M}+\mathrm{H}]^{+}$.

Ethyl 1-p-chloro-phenylsulfonamido-4-oxo-4H-quinolizine-3-carboxylate (6c): Yield 73\%. m.p.: 210$212^{\circ} \mathrm{C} ;{ }^{1} \mathrm{H}-\mathrm{NMR}$ (DMSO-d $): \delta 1.24\left(\mathrm{t}, 3 \mathrm{H}, J=7.6 \mathrm{~Hz}, \mathrm{CH}_{3}\right), 4.18\left(\mathrm{q}, 2 \mathrm{H}, J=7.6 \mathrm{~Hz}, \mathrm{CH}_{2}\right), 7.54$ (s, $1 \mathrm{H}, \mathrm{Ar}-\mathrm{H}), 7.55$ (dd, 1H, $J=7.2 \mathrm{~Hz}, J=6.4 \mathrm{~Hz}, \mathrm{Ar}-\mathrm{H}), 7.68$ (d, 2H, $J=8.8 \mathrm{~Hz}, \mathrm{Ar}-\mathrm{H}), 7.73$ (d, 2H, $J$ $=8.8 \mathrm{~Hz}, \mathrm{Ar}-\mathrm{H}), 7.97-8.05(\mathrm{~m}, 2 \mathrm{H}, \mathrm{Ar}-\mathrm{H}), 9.29(\mathrm{~d}, 1 \mathrm{H}, J=7.2 \mathrm{~Hz}, \mathrm{Ar}-\mathrm{H}) ;{ }^{13} \mathrm{C}-\mathrm{NMR}\left(\mathrm{DMSO}-d_{6}\right): \delta$ 14.6, 60.3, 103.1, 108.5, 118.7, 122.4, 129.7, 129.9, 130.0, 136.6, 138.0, 138.6, 140.1, 146.0, 154.0, 164.5; IR: $v$ 3445, 3155, 2972, 1715, $1494 \mathrm{~cm}^{-1}$; MS (ESI): $m / z 404.9$ [M -1] $]^{-}, 428.8[\mathrm{M}+\mathrm{Na}]^{+}$.

Ethyl 1-p-methyloxyphenylsulfonamido-4-oxo-4H-quinolizine-3-carboxylate (6d): Yield 92\%. m.p.: 236-238 ${ }^{\circ} \mathrm{C} ;{ }^{1} \mathrm{H}$ NMR (DMSO- $\left.d_{6}\right): \delta 1.19\left(\mathrm{t}, 3 \mathrm{H}, J=6.9 \mathrm{~Hz}, \mathrm{CH}_{3}\right), 3.82\left(\mathrm{~s}, 3 \mathrm{H}, \mathrm{OCH}_{3}\right), 4.11(\mathrm{q}, 2 \mathrm{H}, J=$ 7.0, $\left.\mathrm{CH}_{2}\right), 7.06(\mathrm{~d}, 2 \mathrm{H}, J=8.6, \mathrm{Ar}-\mathrm{H}), 7.49-7.51(\mathrm{~m}, 2 \mathrm{H}, \mathrm{Ar}-\mathrm{H}), 7.60(\mathrm{~d}, 2 \mathrm{H}, J=8.6 \mathrm{~Hz}, \mathrm{Ar}-\mathrm{H}), 7.90-$ $8.01(\mathrm{~m}, 2 \mathrm{H}, \mathrm{Ar}-\mathrm{H}), 9.24(\mathrm{~d}, 1 \mathrm{H}, J=7.2 \mathrm{~Hz}, \mathrm{Ar}-\mathrm{H}), 9.58(\mathrm{~s}, 1 \mathrm{H}, \mathrm{NH}) ;{ }^{13} \mathrm{C}$ NMR (DMSO- $\left.d_{6}\right): \delta 14.5$, 56.1, 60.4, 103.2, 109.1, 114.9, 118.7, 122.5, 129.8, 129.9, 130.6, 136.3, 140.3, 146.0, 154.1, 163.2, 164.6; IR: $v 3442,3170,2927,1716,1650,1494 \mathrm{~cm}^{-1}$; MS (ESI): $m / z 424.9[\mathrm{M}+\mathrm{Na}]^{+}$.

Ethyl 1-p-nitrophenylsulfonamido-4-oxo-4H-quinolizine-3-carboxylate (6e): Yield: 76\%. m.p.: 265$267^{\circ} \mathrm{C}$; ${ }^{1} \mathrm{H}-\mathrm{NMR}$ (DMSO- $d_{6}$ ): $\delta 1.15\left(\mathrm{t}, 3 \mathrm{H}, J=7.2 \mathrm{~Hz}, \mathrm{CH}_{3}\right), 4.1$ (q, $2 \mathrm{H}, J=7.2 \mathrm{~Hz}, \mathrm{CH}_{2}$ ), 7.51 (s, 1H, Ar-H), 7.49-7.53 (m, 1H, Ar-H), 7.94-7.98 (m, 4H, Ar-H), 8.39 (d, 2H, J=8.8 Hz, Ar-H), 9.27 (d, $1 \mathrm{H}, J=7.2 \mathrm{~Hz}, \mathrm{Ar}-\mathrm{H}), 10.20$ (s, $1 \mathrm{H}, \mathrm{NH}) ;{ }^{13} \mathrm{C}-\mathrm{NMR}$ (DMSO- $d_{6}$ ): $\delta 14.5,60.4,103.1,108.0,118.8$, 122.2, 125.0, 129.4, 130.1, 136.8, 140.1, 144.8, 145.8, 150.4, 154.1, 164.6; IR: v 3446, 3204, 2981, $1727,1627,1497,1233 \mathrm{~cm}^{-1}$; MS (ESI): $\mathrm{m} / z 439.9[\mathrm{M}+\mathrm{Na}]^{+}$.

General procedure for the synthesis of 1-(substituted phenylsulfonamido)-4-oxo-4H-quinolizine-3carboxylic acids $\mathbf{8}$

To a suspension of $6(1.2 \mathrm{mmol})$ in methanol $(15 \mathrm{~mL})$ was added $3 \mathrm{~mol} / \mathrm{L}$ of $\mathrm{NaOH}(0.9 \mathrm{~mL})$. The reaction mixture was stirred overnight, followed by evaporation of the solvent under reduced pressure. The remaining solid was dissolved in water $(20 \mathrm{~mL})$ and the clear solution was acidified with $1 \mathrm{~mol} / \mathrm{L}$ $\mathrm{HCl}$ to $\mathrm{pH}$ 1. The resulting suspension was filtered and dried under vacuum over $\mathrm{P}_{2} \mathrm{O}_{5}$ to give yellowish solids.

1-Phenylsulfonamido-4-oxo-4H-quinolizine-3-carboxylic acid (8a): Yield 82\%. m.p.: 245-247 ${ }^{\circ}$; ${ }^{1} \mathrm{H}$ NMR (DMSO- $\left.d_{6}\right): \delta 7.51-7.55(\mathrm{~m}, 2 \mathrm{H}, \mathrm{Ar}-\mathrm{H}), 7.63-7.66(\mathrm{~m}, 5 \mathrm{H}, \mathrm{Ar}-\mathrm{H}), 8.00-8.09(\mathrm{~m}, 2 \mathrm{H}, \mathrm{Ar}-\mathrm{H}), 9.29$ $(\mathrm{d}, 1 \mathrm{H}, J=7.2 \mathrm{~Hz}, \mathrm{Ar}-\mathrm{H}), 10.05(\mathrm{~s}, 1 \mathrm{H}, \mathrm{NH}), 13.71$ (s, $1 \mathrm{H}, \mathrm{COOH}) ;{ }^{13} \mathrm{C}-\mathrm{NMR}$ (DMSO- $\left.d_{6}\right): \delta 103.7$, 113.0, 120.2, 122.9, 127.6, 129.8, 133.8, 136.9, 138.6, 138.9, 145.0, 158.9, 165.6; IR: v 3441, 3293, $3116,1727,1616,1495,1446,1365,1327,1159 \mathrm{~cm}^{-1}$; MS (ESI): $m / z 344.9[\mathrm{M}+\mathrm{H}]^{+}, 366.9[\mathrm{M}+$ $\mathrm{Na}]^{+}$.

1-p-Methylphenylsulfonamido-4-oxo-4H-quinolizine-3-carboxylic acid (8b): Yield 75\%. m.p.: > 300 ${ }^{\circ} \mathrm{C}$; ${ }^{1} \mathrm{H}-\mathrm{NMR}$ (DMSO- $\left.d_{6}\right): \delta 2.35\left(\mathrm{~s}, 3 \mathrm{H}, \mathrm{CH}_{3}\right), 7.33(\mathrm{~d}, 2 \mathrm{H}, J=8.1 \mathrm{~Hz}, \mathrm{Ar}-\mathrm{H}), 7.54(\mathrm{~d}, 2 \mathrm{H}, J=8.1 \mathrm{~Hz}$, 
Ar-H), $7.64(\mathrm{dd}, 1 \mathrm{H}, J=7.0 \mathrm{~Hz}, \mathrm{Ar}-\mathrm{H}), 7.67$ (s, 1H, Ar-H), 8.03 (dd, 1H, J=7.2 Hz, J=8.4 Hz, ArH), $8.11(\mathrm{~d}, 1 \mathrm{H}, J=8.8 \mathrm{~Hz}, \operatorname{Ar}-\mathrm{H}), 9.29(\mathrm{~d}, 1 \mathrm{H}, J=7.1 \mathrm{~Hz}, \mathrm{Ar}-\mathrm{H}) ;{ }^{13} \mathrm{C}-\mathrm{NMR}$ (DMSO- $\left.d_{6}\right): \delta 21.5$, 103.7, 113.3, 120.2, 123.0, 127.6, 129.8, 130.2, 136.2, 136.8, 138.5, 144.1, 145.0, 158.9, 165.7; IR: $v$ 3265, 3071, 1725, 1619, 1492, 1448, 1292, 1160, $816 \mathrm{~cm}^{-1}$; HRMS: $m / z$ calcd for $\mathrm{C}_{17} \mathrm{H}_{13} \mathrm{~N}_{2} \mathrm{O}_{5} \mathrm{~S}$ : 357.0551; found: 357.0550 .

1-p-Chlorophenylsulfonamido-4-oxo-4H-quinolizine-3-carboxylic acid (8c): Yield 79\%. $\mathrm{mp}>300^{\circ} \mathrm{C}$; ${ }^{1} \mathrm{H}-\mathrm{NMR}$ (DMSO- $\left.d_{6}\right): \delta$ 7.65-7.72 (m, 6H, Ar-H), 8.09-8.15 (m, 2H, Ar-H), $9.36(\mathrm{~d}, 1 \mathrm{H}, J=7.1 \mathrm{~Hz}$, $\mathrm{Ar}-\mathrm{H}), 10.22(\mathrm{~s}, 1 \mathrm{H}, \mathrm{NH}), 13.70(\mathrm{~s}, 1 \mathrm{H}, \mathrm{OH}) ;{ }^{13} \mathrm{C}-\mathrm{NMR}$ (DMSO- $\left.d_{6}\right): \delta 103.7,112.7,120.3,122.9$, 129.6, 129.9, 129.9, 137.0, 137.8, 138.5, 138.6, 145.0, 158.9, 165.7; IR: v 3446, 3265, 2924, 1720, 1489, $1163 \mathrm{~cm}^{-1}$; MS (ESI): $m / z 376.9$ [M-H].

1-p-Methoxyphenylsulfonamido-4-oxo-4H-quinolizine-3-carboxylic acid (8d): Yield 86\%. mp > $300{ }^{\circ} \mathrm{C} ;{ }^{1} \mathrm{H}-\mathrm{NMR}\left(\mathrm{DMSO}-d_{6}\right): \delta 3.79\left(\mathrm{~s}, 3 \mathrm{H}, \mathrm{CH}_{3}\right), 7.03(\mathrm{~d}, 2 \mathrm{H}, J=8.8 \mathrm{~Hz}, \mathrm{Ar}-\mathrm{H}), 7.57(\mathrm{~d}, 2 \mathrm{H}, J=8.8$ $\mathrm{Hz}, \mathrm{Ar}-\mathrm{H}), 7.65$ (dd, 1H, $J=10.0 \mathrm{~Hz}, J=7.2 \mathrm{~Hz}, \mathrm{Ar}-\mathrm{H}), 7.68$ (s, 1H, Ar-H), 8.03 (dd, 1H, $J=8.6 \mathrm{~Hz}$, $J=7.0, \operatorname{Ar}-\mathrm{H}), 8.10(\mathrm{~d}, 1 \mathrm{H}, J=8.8 \mathrm{~Hz}, \mathrm{Ar}-\mathrm{H}), 9.29$ (d, $1 \mathrm{H}, J=7.2 \mathrm{~Hz}, \mathrm{Ar}-\mathrm{H}), 9.86(\mathrm{~s}, 1 \mathrm{H}, \mathrm{NH}) ;{ }^{13} \mathrm{C}-$ NMR (DMSO- $d_{6}$ ): $\delta 56.2,103.7,113.3,114.9,120.2,123.0,129.7,129.8,130.5,136.8,138.6,145.1$, 158.9, 163.3, 165.7; IR: $v$ 3248, 2918, 2849, 1724, 1618, 1597, 1496, 1450, 1294, 1157, $836 \mathrm{~cm}^{-1}$; MS (ESI): $m / z 375.0[\mathrm{M}+\mathrm{H}]^{+}$.

1-p-Nitrophenylsulfonamido-4-oxo-4H-quinolizine-3-carboxylic acid $(\mathbf{8 e})$ : Yield $82 \% ; \mathrm{mp}>300^{\circ} \mathrm{C}$; ${ }^{1} \mathrm{H}-\mathrm{NMR}$ (DMSO- $\left.d_{6}\right): \delta 7.60-7.80(\mathrm{~m}, 1 \mathrm{H}, \mathrm{Ar}-\mathrm{H}), 7.73(\mathrm{~s}, 1 \mathrm{H}, \mathrm{Ar}-\mathrm{H}), 7.94(\mathrm{~d}, 2 \mathrm{H}, J=8.0 \mathrm{~Hz}, \mathrm{Ar}-\mathrm{H})$, 8.02-8.05 (m, 2H, Ar-H), 8.35 (d, 2H, J=8.0 Hz, Ar-H), 9.32 (d, 1H, J=6.4 Hz, Ar-H), 10.49 (s, 1H, $\mathrm{NH}), 13.68(\mathrm{~s}, 1 \mathrm{H}, \mathrm{OH}) ;{ }^{13} \mathrm{C}-\mathrm{NMR}$ (DMSO- $\left.d_{6}\right): \delta 103.8,112.3,120.3,122.6,125.1,129.3,129.9$, 137.2, 138.6, 144.7, 144.8, 150.4, 158.9, 165.7; IR: $v 3454,3234,3082,1715,1488,1167,786 \mathrm{~cm}^{-1}$; MS (ESI): $m / z 387.8$ [M-H]'.

\section{General procedure for the synthesis of $\mathbf{7 a , b}$}

To a solution of 3,4,5-trimethoxylbenzoic acid or 3,4-dimethoxycinnamic acid (4 mmol) in ether $(20 \mathrm{~mL})$ was added $\mathrm{PCl}_{5}(20 \mathrm{~mL})$ in one portion. The reaction mixture was heated to reflux for about 4 hours and then cooled to room temperature. After maintaining in a refrigerator for about 1 hour, a white precipitate formed which was filtered and recrystallized from cold ether to give the corresponding carboxylic chloride. The newly-synthesized acyl chloride dissolved in $\mathrm{CH}_{2} \mathrm{Cl}_{2}(20 \mathrm{~mL})$ was added dropwise to a mixture of compound $5(3 \mathrm{mmol})$ and triethylamine $(3 \mathrm{mmol})$ in $\mathrm{CH}_{2} \mathrm{Cl}_{2}(20$ $\mathrm{mL}$ ). The reaction solution was stirred overnight at room temperature. After the reaction, the solution was washed with water and the organic layer was dried over anhydrous $\mathrm{MgSO}_{4}$. After filtration of the $\mathrm{MgSO}_{4}$, the solvent was removed under reduced pressure and the residue was purified by recrystallization from ethyl acetate to give $\mathbf{7 a}$ or $\mathbf{7 b}$ as a yellow solid.

Ethyl 1-(3,4,5-trimethoxyphenylcarbonylamino)-4-oxo-4H-quinolizine-3-carboxylate (7a): Yield 40\%; mp 93 ${ }^{\circ} \mathrm{C} ;{ }^{1} \mathrm{H}-\mathrm{NMR}\left(\mathrm{CDCl}_{3}\right): \delta 1.29\left(\mathrm{t}, 3 \mathrm{H}, J=7.2 \mathrm{~Hz}, \mathrm{CH}_{3}\right), 3.92\left(\mathrm{~s}, 3 \mathrm{H}, \mathrm{OCH}_{3}\right), 3.95\left(\mathrm{~s}, 6 \mathrm{H}, \mathrm{OCH}_{3}\right)$, 
$4.26\left(\mathrm{q}, 2 \mathrm{H}, J=7.2 \mathrm{~Hz}, \mathrm{OCH}_{2}\right), 7.11(\mathrm{dd}, 1 \mathrm{H}, J=6.8 \mathrm{~Hz}, J=7.2 \mathrm{~Hz}, \mathrm{Ar}-\mathrm{H}), 7.39$ (s, 2H, Ph-H), 7.57 (dd, $1 \mathrm{H}, J=8.8 \mathrm{~Hz}, J=6.8 \mathrm{~Hz}, \mathrm{Ar}-\mathrm{H}), 7.66$ (d, 1H, $J=8.8 \mathrm{~Hz}, \mathrm{Ar}-\mathrm{H}), 8.08$ (s, 1H, Ar-H), 8.66 (bs, $1 \mathrm{H}, \mathrm{NH}), 9.17(\mathrm{~d}, 1 \mathrm{H}, J=7.2 \mathrm{~Hz}, \mathrm{Ar}-\mathrm{H}) ;{ }^{13} \mathrm{C}-\mathrm{NMR}\left(\mathrm{CDCl}_{3}\right): \delta=14.4,56.4,60.9,61.0,105.1,105.1$, 110.6, 116.9, 122.0, 128.4, 129.4, 134.2, 140.2, 141.5, 143.7, 153.3, 154.9, 165.3, 166.9; IR: v 3462, $3328,2939,1720,1678,1587,1487,1336,1216,1126,1009,772 \mathrm{~cm}^{-1}$; MS (ESI): $\mathrm{m} / z 449.0[\mathrm{M}+$ $\mathrm{Na}]^{+}$.

Ethyl 1-[3-(3,4-dimethoxyphenyl)acrylamido]-4-oxo-4H-quinolizine-3-carboxylate (7b): Yield 85\%; m.p.: $105-107^{\circ} \mathrm{C} ;{ }^{1} \mathrm{H}-\mathrm{NMR}$ (DMSO- $\left.d_{6}\right): \delta 1.30$ (t, $3 \mathrm{H}, J=7.2 \mathrm{~Hz}, \mathrm{CH}_{3}$ ), $3.81\left(\mathrm{~s}, 3 \mathrm{H}, \mathrm{OCH}_{3}\right), 3.83$ (s, $\left.3 \mathrm{H}, \mathrm{OCH}_{3}\right), 4.26\left(\mathrm{q}, 2 \mathrm{H}, \mathrm{J}=7.2 \mathrm{~Hz}, \mathrm{CH}_{2}\right), 6.81$ (d, $\left.1 \mathrm{H}, J=15.6 \mathrm{~Hz}, \mathrm{CH}=\mathrm{CH}\right), 7.03(\mathrm{~d}, 1 \mathrm{H}, J=8.4 \mathrm{~Hz}$, Ar-H), 7.21 (d, 1H, J=8.4 Hz, Ar-H), 7.26 (s, 1H, Ar-H), 7.51 (dd, 1H, J=6.4 Hz, J=7.2 Hz, Ar-H), $7.55(\mathrm{~d}, 1 \mathrm{H}, J=15.6 \mathrm{~Hz}, \mathrm{Ar}-\mathrm{H}), 7.86(\mathrm{~d}, 1 \mathrm{H}, \mathrm{J}=8.8 \mathrm{~Hz}, \mathrm{Ar}-\mathrm{H}), 7.96(\mathrm{dd}, 1 \mathrm{H}, J=7.2 \mathrm{~Hz}, J=8.0 \mathrm{~Hz}$, Ar-H), 8.26 (s, 1H, Ar-H), 9.30 (d, $1 \mathrm{H}, J=7.2 \mathrm{~Hz}, \mathrm{Ar}-\mathrm{H}), 9.89$ (s, $1 \mathrm{H}, \mathrm{NH}) ;{ }^{13} \mathrm{C}-\mathrm{NMR}$ (DMSO- $\left.d_{6}\right): \delta$ $14.8,55.9,56.0,60.5,103.7,110.6,111.1,112.2,118.2,119.4,122.3,122.4,127.9,129.8,135.8$, 139.0, 141.1, 143.1, 149.4, 150.9, 154.1, 165.3, 165.9; IR: v 3436, 3226, 2935, 1702, 1677, 1595, $1491,1377,1236,1114,1026,768 \mathrm{~cm}^{-1}$; MS (ESI): $m / z 445.0[\mathrm{M}+\mathrm{Na}]^{+}$.

\section{General procedure for the synthesis of $\mathbf{9 a , b}$}

To a suspension of $\mathbf{7 a}$ or $\mathbf{7 b}(0.5 \mathrm{mmol})$ in $80 \%$ methanol $(20 \mathrm{~mL})$ was added $\mathrm{NaOH}(5 \mathrm{mmol})$. The reaction mixture was heated to reflux till the starting $\mathbf{7 a}$ or $\mathbf{7 b}$ disappeared according to monitoring by TLC. After evaporation of the solvent under reduced pressure, the remaining solid was dissolved in water $(20 \mathrm{~mL})$ and the clear solution was acidified with $1 \mathrm{~mol} / \mathrm{L} \mathrm{HCl}$ to $\mathrm{pH} 1$ and the resulting suspension extracted with $\mathrm{CH}_{2} \mathrm{Cl}_{2}$. The combined organic layer was dried over anhydrous $\mathrm{MgSO}_{4}$ followed by filtration. The desired $\mathbf{9 a}$ or $\mathbf{9 b}$ were finally obtained as solids by column chromatography (eluents: $\mathrm{CH}_{3} \mathrm{OH} / \mathrm{CHCl}_{3}=1: 30$ )

1-(3,4,5-Trimethoxyphenylcarbonylamino)-4-oxo-4H-quinolizine-3-carboxylic acid (9a): Yield 92\%; m.p.: $280-282^{\circ} \mathrm{C}$; ${ }^{1} \mathrm{H}-\mathrm{NMR}$ (DMSO-d ${ }_{6}$ ): $\delta 3.67$ (s, 3H, $\left.\mathrm{OCH}_{3}\right), 3.80\left(\mathrm{~s}, 6 \mathrm{H}, \mathrm{OCH}_{3}\right), 7.33(\mathrm{~s}, 2 \mathrm{H}, \mathrm{Ar}-\mathrm{H})$, $7.64(\mathrm{dd}, 1 \mathrm{H}, J=7.2 \mathrm{~Hz}, J=6.8 \mathrm{~Hz}, \mathrm{Ar}-\mathrm{H}), 7.94(\mathrm{~d}, 1 \mathrm{H}, J=8.8 \mathrm{~Hz}, \mathrm{Ar}-\mathrm{H}), 8.02$ (dd, 1H, $J=6.8 \mathrm{~Hz}, J$ $=8.8 \mathrm{~Hz}, \mathrm{Ar}-\mathrm{H}$ ), 8.29 (s, 1H, Ar-H), 9.30 (d, 1H, J=7.2 Hz, Ar-H), 10.29 (s, 1H, NH), 13.96 (s, 1H, $\mathrm{COOH}) ;{ }^{13} \mathrm{C}-\mathrm{NMR}$ (DMSO- $d_{6}$ ): $\delta 56.6,60.6,103.9,105.9,115.2,105.9,115.2,120.2,123.3,129.1$, 129.7, 136.6, 138.2, 141.0, 143.0, 153.2, 159.0, 166.1, 166.5; IR: v 3440, 3115, 2924, 2853, 1696, $1657,1615,1587,1496,1453,1364,1292,1123 \mathrm{~cm}^{-1}$; MS (ESI): $m / z 397.0$ [M - H] .

1-(3,4-Dimethoxystyrylcarboxylamino)-4-oxo-4H-quinolizine-3-carboxylic acid (9b): Yield 91\%; m.p.: 306-308 ${ }^{\circ} \mathrm{C}$; ${ }^{1} \mathrm{H}-\mathrm{NMR}$ (DMSO-d $\left.d_{6}\right): \delta 3.82\left(\mathrm{~s}, 3 \mathrm{H}, \mathrm{OCH}_{3}\right), 3.84\left(\mathrm{~s}, 3 \mathrm{H}, \mathrm{OCH}_{3}\right), 6.84(\mathrm{~d}, 1 \mathrm{H}, J=16.0 \mathrm{~Hz}$, $\mathrm{CH}=\mathrm{CH}), 7.03(\mathrm{~d}, 1 \mathrm{H}, J=8.4 \mathrm{~Hz}, \mathrm{Ar}-\mathrm{H}), 7.23(\mathrm{~d}, 1 \mathrm{H}, J=8.4 \mathrm{~Hz}, \mathrm{Ar}-\mathrm{H}), 7.27(\mathrm{~s}, 1 \mathrm{H}, \mathrm{Ar}-\mathrm{H}), 7.58(\mathrm{~d}$, $1 \mathrm{H}, J=16.0 \mathrm{~Hz}, \mathrm{Ar}-\mathrm{H}), 7.70-7.74(\mathrm{~m}, 1 \mathrm{H}, \mathrm{Ar}-\mathrm{H}), 8.10-8.12$ (m, 2H, Ar-H), 8.44 (s, 1H, Ar-H), 9.37 (d, $1 \mathrm{H}, J=7.2 \mathrm{~Hz}, \mathrm{Ar}-\mathrm{H}$ ), 10.11 (s, $1 \mathrm{H}, \mathrm{NH}), 14.13$ (s, $1 \mathrm{H}, \mathrm{OH}) ;{ }^{13} \mathrm{C}-\mathrm{NMR}$ (DMSO-d $)_{6}$ : $\delta 56.0,56.1$, $103.9,110.9,112.4,115.5,119.3,120.0,122.3,122.8,127.9,136.3,136.9,141.4,142.1,149.5,151.1$, 
158.8, 165.8, 166.1; IR: $v 3251,1728,1612,1541,1515,1464,1303,1266 \mathrm{~cm}^{-1}$; MS (ESI): $\mathrm{m} / z 393.1$ $[\mathrm{M}-\mathrm{H}]^{-}$.

Synthesis of ethyl 1-benzothiazol-2-yl-4-oxo-4H-quinolizine-3-carboxylate (11)

2-Aminophenthiol (250 $\mathrm{mg}, 2 \mathrm{mmol}$ ) was added to a $50 \mathrm{~mL}$ of round flask charged with compound 10 (490 mg, $2 \mathrm{mmol})$ and acetic acid $(15 \mathrm{~mL})$. The reaction mixture was heated to reflux for about one hour till TLC confirmed that the reaction had finished. After removal of the solvent, the residue was recrystallized from ethyl acetate to offer a needle-like yellow solid. Yield: 65\%; m.p.: 199-200 ${ }^{\circ} \mathrm{C}$; ${ }^{1} \mathrm{H}-\mathrm{NMR}$ (DMSO-d $\left.d_{6}\right): \delta 1.35\left(\mathrm{t}, 3 \mathrm{H}, J=7.1 \mathrm{~Hz}, \mathrm{CH}_{2} \mathrm{CH}_{3}\right), 4.32\left(\mathrm{q}, 2 \mathrm{H}, J=7.1 \mathrm{~Hz}, \mathrm{CH}_{2} \mathrm{CH}_{3}\right), 7.48(\mathrm{t}$, $1 \mathrm{H}, J=7.6 \mathrm{~Hz}, \operatorname{Ar}-H), 7.57$ (t, $1 \mathrm{H}, J=7,6 \mathrm{~Hz}, \operatorname{Ar}-H), 7.70$ (t, $1 \mathrm{H}, J=7.2 \mathrm{~Hz}), 8.07$ (d, $1 \mathrm{H}, J=8.0 \mathrm{~Hz}$, $\operatorname{Ar}-H), 8.15(\mathrm{~d}, 1 \mathrm{H}, J=8.0 \mathrm{~Hz}, \operatorname{Ar}-H), 8.25(\mathrm{t}, 1 \mathrm{H}, J=7.6 \mathrm{~Hz}, \operatorname{Ar}-H), 8.75(\mathrm{~s}, 1 \mathrm{H}, \operatorname{Ar}-H), 9.47(\mathrm{~d}, 1 \mathrm{H}$, $J=7.2 \mathrm{~Hz}, \mathrm{Ar}-H$ ), $9.59(\mathrm{~d}, 1 \mathrm{H}, J=8.0 \mathrm{~Hz}, \mathrm{Ar}-H) ;{ }^{13} \mathrm{C}-\mathrm{NMR}$ (DMSO-d $\left.)\right): \delta 15.2,61.2,105.0,106.0$, 120.0, 122.8, 123.3, 124.9, 126.1, 127.5, 131.3, 134.2, 139.4, 142.9, 144.6, 154.3, 154.7, 165.4, 165.9; IR: $v 1736,1670,1623,1583,1496,1222,1133,780 \mathrm{~cm}^{-1}$; MS (ESI): $\mathrm{m} / z 373[\mathrm{M}+\mathrm{Na}]^{+}$.

Synthesis of 1-benzothiazol-2-yl-4-oxo-4H-quinolizine-3-carboxylic acid (12)

$\mathrm{KOH}(765 \mathrm{mg}, 13.7 \mathrm{mmol})$ dissolved in water $(6 \mathrm{~mL})$ was added to a $100 \mathrm{~mL}$ round flask charged with compound $11(480 \mathrm{mg}, 1.37 \mathrm{mmol})$ and ethanol $(24 \mathrm{~mL})$. The reaction mixture was heated to reflux for about four hours till TLC confirmed that the reaction had finished. After removal of the solvent, the residue was re-dissolved in water $(30 \mathrm{~mL})$ and then acidified to $\mathrm{pH} 2$. The product was extracted three times with $\mathrm{CH}_{2} \mathrm{Cl}_{2}(50 \mathrm{~mL})$ and the combined organic layer was dried over anhydrous $\mathrm{MgSO}_{4}$. After filtration of the $\mathrm{MgSO}_{4}$, the solvent was removed under reduced pressure and the residue was purified by recrystallization from chloroform/petroleum ether to generate $\mathbf{1 2}$ as a yellow solid. Yield 68\%. m.p.: $>300{ }^{\circ} \mathrm{C} ;{ }^{1} \mathrm{H}-\mathrm{NMR}\left(\mathrm{CDCl}_{3}\right): \delta 7.32(\mathrm{dd}, 1 \mathrm{H}, J=7.6 \mathrm{~Hz}, J=7.2 \mathrm{~Hz}, \mathrm{Ar}-H)$, 7.64-7.67 (m, 2H, Ar-H), 7.81-7.91(m, 2H, Ar-H), 7.95 (d, 1H, $J=8.0 \mathrm{~Hz}, \operatorname{Ar}-H), 8.78(\mathrm{~d}, 1 \mathrm{H}, J=4.8$ $\mathrm{Hz}), 9.21(\mathrm{~s}, 1 \mathrm{H}, \mathrm{Ar}-H), 9.43(\mathrm{~d}, 1 \mathrm{H}, J=7.2 \mathrm{~Hz}, \mathrm{Ar}-H), 13.95(\mathrm{~s}, 1 \mathrm{H}, \mathrm{COO} H) ;{ }^{13} \mathrm{C}-\mathrm{NMR}$ (DMSO- $\left.d_{6}\right)$ : $\delta 110.2,113.2,119.9,120.6,122.6,123.3,127.6,128.3,130.7,137.3,138.3,138.7,147.7,151.4$, 152.4, 163.8, 165.7; IR: $v$ 1750, 1611, 1564, $1453 \mathrm{~cm}^{-1}$; MS (ESI): $m / z 345.0[\mathrm{M}+\mathrm{Na}]^{+}, 361.0[\mathrm{M}+$ $\mathrm{K}]^{+}$.

General procedure for the synthesis of compounds 13a-c

To a $50 \mathrm{~mL}$ of round flask charged with phenylenediamine derivative $(1 \mathrm{mmol})$ and nitrobenzene $(10 \mathrm{~mL})$ was added compound $\mathbf{1 0}(245 \mathrm{mg}, 1 \mathrm{mmol})$ and then the solution was slowly heated to $100{ }^{\circ} \mathrm{C}$. After $1 \mathrm{~h}$ stirring, the reaction mixture was further heated to $150{ }^{\circ} \mathrm{C}$ and stirred till TLC showed the absence of starting 10. The solution was cooled to room temperature and directly loaded onto a silica column for chromatographic purification. The eluents were first petroleum ether and then ethyl acetate/petroleum ether (1:2). After the nitrobenzene was eluted out, $\mathrm{CHCl}_{3} / \mathrm{CH}_{3} \mathrm{OH}$ was used to elute the corresponding 13a-c. 
Ethyl 1-(1H-benzimidazol-2-yl)-4-oxo-4H-quinolizine-3-carboxylate (13a): Yield 85 \%. m.p.: 194-195 ${ }^{\circ} \mathrm{C} ;{ }^{1} \mathrm{H}-\mathrm{NMR}\left(\mathrm{CDCl}_{3}\right): \delta 1.20\left(\mathrm{t}, 3 \mathrm{H}, J=7.2 \mathrm{~Hz}, \mathrm{OCH}_{2} \mathrm{CH}_{3}\right), 4.12$ (q, 2H, $\left.J=7.2 \mathrm{~Hz}, \mathrm{OCH}_{2} \mathrm{CH}_{3}\right), 7.21$ (t, $1 \mathrm{H}, J=7.2 \mathrm{~Hz}, \mathrm{Ar}-H), 7.31 \sim 7.36(\mathrm{~m}, 2 \mathrm{H}, \mathrm{Ar}-H), 7.67 \sim 7.75$ (m, 3H, Ar-H), 8.56 (s, 1H, Ar-H), 8.94 $(\mathrm{d}, 1 \mathrm{H}, J=8.9 \mathrm{~Hz}, \mathrm{Ar}-H), 9.30(\mathrm{~d}, 1 \mathrm{H}, J=7.2 \mathrm{~Hz}), 11.5(\mathrm{br}, 1 \mathrm{H}, \mathrm{N} H) ;{ }^{13} \mathrm{C}-\mathrm{NMR}\left(\mathrm{CDCl}_{3}\right): \delta 14.2$, 61.1, 104.8, 105.2, 117.7, 122.9, 124.8, 129.6, 135.6, 141.1, 144.6, 148.8, 155.2, 165.3; IR: $v 1725$, $1678,1624,1538 \mathrm{~cm}^{-1}$; MS (ESI): $m / z 334[\mathrm{M}+1], 356[\mathrm{M}+\mathrm{Na}]^{+}$.

Ethyl 1-(5(6)-chloro-1H-benzimidazol-2-yl)-4-oxo-4H-quinolizine-3-carboxylate (13b): Yield $83 \%$; m.p.: 216-217 ${ }^{\circ} \mathrm{C}$; ${ }^{1} \mathrm{H}-\mathrm{NMR}\left(\mathrm{CDCl}_{3}\right): \delta 1.18$ (t, 3H, $\left.J=7.2 \mathrm{~Hz}, \mathrm{OCH}_{2} \mathrm{CH}_{3}\right), 4.10$ (q, 2H, $J=7.2 \mathrm{~Hz}$, $\left.\mathrm{OCH}_{2} \mathrm{CH}_{3}\right), 7.23-7.29(\mathrm{~m}, 2 \mathrm{H}, \mathrm{Ar}-H), 7.63-7.76(\mathrm{~m}, 3 \mathrm{H}, \mathrm{Ar}-H), 8.57(\mathrm{~s}, 1 \mathrm{H}, \mathrm{Ar}-H), 9.04(\mathrm{~d}, 1 \mathrm{H}, J=$ $8.8 \mathrm{~Hz}, \mathrm{Ar}-H), 9.33(\mathrm{~d}, 1 \mathrm{H}, J=4.8 \mathrm{~Hz}, \mathrm{Ar}-H), 11.8$ (br, $1 \mathrm{H}, \mathrm{N} H) ;{ }^{13} \mathrm{C}-\mathrm{NMR}\left(\mathrm{CDCl}_{3}\right): \delta 14.1,61.0$, $104.4,104.9,117.9,123.3,124.7,128.3,129.6,135.8,141.1,144.5,150.0,155.1,165.1$; IR: $v$ 3444, $3219,1730,1644,1488,1106 \mathrm{~cm}^{-1}$; MS (ESI): $\mathrm{m} / z 366$ [M-1]'.

Ethyl 1-(5(6)-methoxy-1H-benzimidazol-2-yl)-4-oxo-4H-quinolizine-3-carboxylate (13c): Yield 88\%; m.p.: $234-236{ }^{\circ} \mathrm{C}$; ${ }^{1} \mathrm{H}-\mathrm{NMR}\left(\mathrm{CDCl}_{3}\right): \delta 1.20\left(\mathrm{t}, 3 \mathrm{H}, J=7.2 \mathrm{~Hz}, \mathrm{OCH}_{2} \mathrm{CH}_{3}\right), 3.89\left(\mathrm{~s}, 3 \mathrm{H}, \mathrm{OCH}_{3}\right), 4.13$ $\left(\mathrm{q}, 2 \mathrm{H}, J=7.2 \mathrm{~Hz}, \mathrm{OCH}_{2} \mathrm{CH}_{3}\right), 6.95(\mathrm{~d}, 1 \mathrm{H}, J=8.8, \mathrm{Ar}-H), 7.21-7.71(\mathrm{~m}, .3 \mathrm{H}, \mathrm{Ar}-H), 8.24(\mathrm{~d}, 1 \mathrm{H}, J=$ $8.0 \mathrm{~Hz}, \operatorname{Ar}-H), 8.54$ (s, $1 \mathrm{H}, \operatorname{Ar}-H), 8.95$ (d, $1 \mathrm{H}, J=8.8 \mathrm{~Hz}, \operatorname{Ar}-H), 9.30$ (d, $1 \mathrm{H}, \mathrm{d}, J=7.2 \mathrm{~Hz}, \operatorname{Ar}-H)$; ${ }^{13} \mathrm{C}-\mathrm{NMR}\left(\mathrm{CDCl}_{3}\right): \delta 165.36,156.81,155.19,144.55,140.98,135.40,134.56,129.53,129.30,124.83$, 123.49, 117.66, 105.13, 105.08, 61.05, 55.85, 14.18; IR: $v 3435,1723,1615,1450 \mathrm{~cm}^{-1}$; MS (ESI): $\mathrm{m} / \mathrm{z}$ $362[\mathrm{M}-1]^{-}$.

General procedure for the synthesis of compounds $\mathbf{1 4 a - c}$

To a $100 \mathrm{~mL}$ of round flask charged with compound $13(1 \mathrm{mmol})$ and ethanol $(20 \mathrm{~mL})$ was added $\mathrm{KOH}(560 \mathrm{mg}, 10 \mathrm{mmol})$ dissolved in water $(5 \mathrm{~mL})$. The reaction mixtures were heated to reflux till TLC confirmed that the reactions had finished. After removal of the solvent, the residue was redissolved in water $(20 \mathrm{~mL})$ and then acidified to $\mathrm{pH}$ 2. The resulting suspensions were filtered, dried and purified by column chromatography (eluents: $\mathrm{CHCl}_{3} / \mathrm{CH}_{3} \mathrm{OH}$ ) to obtain corresponding 14a-c as yellow solids.

1-(1H-Benzimidazol-2-yl)-4-oxo-4H-quinolizine-3-carboxylic acid (14a): Yield $20 \%$ m.p.: >300 ${ }^{\circ} \mathrm{C}$; ${ }^{1} \mathrm{H}-\mathrm{NMR}$ (DMSO- $\left.d_{6}\right): \delta 7.23(\mathrm{~m}, 2 \mathrm{H}, \mathrm{Ar}-H), 7.53(\mathrm{~m}, 1 \mathrm{H}, \mathrm{Ar}-H), 7.70(\mathrm{~m}, 1 \mathrm{H}, \mathrm{Ar}-H), 7.79(\mathrm{t}, 1 \mathrm{H}, J=$ $6.8 \mathrm{~Hz}, \operatorname{Ar}-H), 8.28$ (t, $1 \mathrm{H}, J=5.6 \mathrm{~Hz}, \operatorname{Ar}-H), 8.97$ (s, 1H, Ar-H), 9.47 (d, $1 \mathrm{H}, J=6.8 \mathrm{~Hz}, \mathrm{Ar}-H), 9.85$ $(\mathrm{d}, 1 \mathrm{H}, J=9.2 \mathrm{~Hz}, \mathrm{Ar}-H), 13.14(\mathrm{~s}, 1 \mathrm{H}, \mathrm{N} H), 13.75$ (s, $1 \mathrm{H}, \mathrm{COOH}) ;{ }^{13} \mathrm{C}-\mathrm{NMR}$ (DMSO- $\left.d_{6}\right): \delta 104.6$, 107.0, 111.8, 119.2, 120.6, 122.3, 123.3, 125.8, 130.2, 134.8, 138.1, 139.4, 143.9, 144.2, 149.1, 159.4, 166.1; IR: $v$ 1705, 1612, 1497, $1443 \mathrm{~cm}^{-1}$; HRMS: $m / z$ calcd for $\mathrm{C}_{17} \mathrm{H}_{10} \mathrm{~N}_{3} \mathrm{O}_{3}$ : 304.0718; found: 304.0727 .

1-(5(6)-Chloro-1H-benzimidazol-2-yl)-4-oxo-4H-quinolizine-3-carboxylic acid (14b): Yield: $35 \%$. m.p. $>300{ }^{\circ} \mathrm{C} ;{ }^{1} \mathrm{H}-\mathrm{NMR}\left(\mathrm{DMSO}-d_{6}\right) \delta: 7.43(\mathrm{dd}, 1 \mathrm{H}, J=8.4 \mathrm{~Hz}, J=1.6 \mathrm{~Hz}, \mathrm{Ar}-H), 7.74(\mathrm{~d}, 1 \mathrm{H}, J=8.4$ Hz, Ar-H), 7.78 (s, 1H, Ar-H), 7.79 (t, $1 \mathrm{H}, J=7.2 \mathrm{~Hz}, \operatorname{Ar}-\mathrm{H}), 8.25$ (t, 1H, J=7.6 Hz, Ar- H), 8.89 (s, 
$1 \mathrm{H}, \operatorname{Ar}-H), 9.09(\mathrm{~d}, 1 \mathrm{H}, J=8.8 \mathrm{~Hz}, \operatorname{Ar}-H), 9.45(\mathrm{~d}, 1 \mathrm{H}, J=7.2 \mathrm{~Hz}, \mathrm{Ar}-H) ;{ }^{13} \mathrm{C}-\mathrm{NMR}$ (DMSO- $\left.d_{6}\right) \delta$ : $101.9,104.9,114.6,116.3,120.7,124.9,125.2,129.2,130.6,133.9,136.0,138.9,141.7,144.2,148.6$, 158.6, 165.7; IR: $v 3434,1718,1612,1499 \mathrm{~cm}^{-1}$; MS (ESI) $\mathrm{m} / \mathrm{z}: 338$ [M-1] .

1-(5(6)-Methoxy-1H-benzimidazol-2-yl)-4-oxo-4H-quinolizine-3-carboxylic acid (14c): Yield $45 \%$. m.p.: $>300{ }^{\circ} \mathrm{C}$; ${ }^{1} \mathrm{H}-\mathrm{NMR}$ (DMSO- $\left.d_{6}\right): \delta 3.86\left(\mathrm{~s}, 3 \mathrm{H}, \mathrm{OCH}_{3}\right), 7.06(\mathrm{dd}, 1 \mathrm{H}, J=8.4 \mathrm{~Hz}, J=2.0 \mathrm{~Hz}, \mathrm{Ar}-$ $H), 7.21(\mathrm{~d}, 1 \mathrm{H}, J=2.0 \mathrm{~Hz}, \operatorname{Ar}-H), 7.66(\mathrm{~d}, 1 \mathrm{H}, J=8.8 \mathrm{~Hz}, \operatorname{Ar}-H), 7.80(\mathrm{t}, 1 \mathrm{H}, J=6.8 \mathrm{~Hz}, \operatorname{Ar}-H), 8.26$ (t, $1 \mathrm{H}, J=8.4 \mathrm{~Hz}, \operatorname{Ar}-H), 8.88(\mathrm{~s}, 1 \mathrm{H}, \operatorname{Ar}-H), 9.09$ (d, $1 \mathrm{H}, J=8.8 \mathrm{~Hz}, \operatorname{Ar}-H), 9.48$ (d, $1 \mathrm{H}, J=7.2 \mathrm{~Hz}$, Ar- $H$ ); ${ }^{13} \mathrm{C}-\mathrm{NMR}$ (DMSO- $d_{6}$ ): $\delta 165.76,158.73,157.63,146.81,144.11,141.15,138.64,136.03$, 130.49, 129.85, 125.03, 120.65, 115.89, 114.55, 104.84, 103.86, 97.09, 56.24; IR: v 3415, 1728, 1618 $\mathrm{cm}^{-1}$; MS (ESI): $m / z 334$ [M-1].

\section{Acknowledgements}

This work was supported by Grants from Beijing Novel Project (No. 2005B10), Beijing Natural Science Foundation (No. 2062003) and the National Natural Science Foundation of China (No. 20772010).

\section{References}

1. Turner, B.G.; Summers, M.F. Structural biology of HIV. J. Mol. Biol. 1999, 285, 1-32.

2. Molla, A.; Granneman, G.R.; Sun, E.; Kempf, D.J. Recent developments in HIV protease inhibitor therapy. Antiviral Res. 1998, 39, 1-23.

3. Moyle, G.; Gazzard, B. Current knowledge and future prospects for the use of HIV protease inhibitors. Drugs 1996, 51, 701-702.

4. Pomerantz, R.J.; Horn, D.L. Twenty years of therapy for HIV 1 infection. Nature Med. 2003, 9, 867-873.

5. De Clercq, E. New developments in anti-HIV chemotherapy. Biochim. Biophys. Acta 2002, 1587, 258-275.

6. Brown, P.O. Integration of retroviral DNA. Curr. Top. Microbiol. Immunol. 1990, 157, 19-48.

7. Katz, R.A.; Skalka, A.M. The retroviral enzymes. Annu. Rev. Biochem. 1994, 63, 133-173.

8. Farnet, C.M.; Wang, B.; lipford, J.R.; Bushman, F.D. Differential inhibition of HIV-1 preintegration complexes and purified integrase protein by small molecules. Proc. Natl. Acad. Sci. USA 1996, 93, 9742-9747.

9. Molteni, V.; Rhodes, D.; Rubins, K.; Hansen, M.; Bushman, F.D.; Siegel, J.S. A new class of HIV-1 integrase inhibitors: the 3,3,3',3'-tetramethyl-1,1'-spirobi(indan)-5,5',6,6'-tetrol family. $J$. Med. Chem. 2000, 43, 2031-2039.

10. Hazuda, D.J.; Felock, P.; Witmer, M.; Wolfe, A.; Stillmock, K.; Grobler, J.A.; Espeseth, A.; Gabryelski, L.; Schleif, W.; Blau, C.; Miller, M.D. Inhibitors of strand transfer that prevent integration and inhibit HIV-1 replication in cells. Science 2000, 287, 646-650.

11. Hazuda, D.J.; Yong, S.D.; Guare, J.P.; Anthony, N.J.; Gomez, R.P.; Wai, J.S.; Vacca, J.P.; Handt, L.; Motzel, S.L.; Klein, H.J.; Tussey, L.; Schleif, W.A.; Gabryelski, L.S.; Jin, L.; Miller, M.D.; 
Casimiro, D.R.; Emini, E.A.; Shiver, J.W. Integrase inhibitors and cellular immunity suppress retroviral replication in rhesus macaques. Science 2004, 305, 528-532.

12. Espeseth, A.S.; Felock, P.; Wolfe, A.; Witmer, M.; Grobler, J.; Anthony, N.; Egbertson, M.; Melamed, J.Y.; Young, S.; Hamill, T.; Cole, J.L.; Hazuda, D.J. HIV-1 integrase inhibitors that compete with the target DNA substrate define a unique strand transfer conformation for integrase. Proc. Natl. Acad. Sci. USA 2000, 97, 11244-11249.

13. Goldgur, Y.; Craigie, R.; Cohen, G.H.; Fujiwara, T.; Yoshinaga, T.; Fujishita, T.; Sugimoto, H.; Endo, T.; Murai, H.; Davies, D.R. Structure of the HIV-1 integrase catalytic domain complexed with an inhibitor: a platform for antiviral drug design. Proc. Natl. Acad. Sci. USA 1999, 96, 13040-13043.

14. Grobler, J.A.; Stillmock, K.; Hu, B.; Witmer, M.; Felock, P.; Espeseth, A.; Wolfe, A.; Egbertson, M.S.; Bourgeois, M.; Melamed, J.Y.; Wai, J.S.; Young, S.D.; Vacca, J.P.; Hazuda, D.J. Diketo acid inhibitor mechanism and HIV-1 integrase: implications for metal binding in the active site of phosphotransferase enzymes. Proc. Natl. Acad. Sci. USA 2002, 99, 6661-6666.

15. Zhuang, L.H.; Wai, J.S.; Embrey, M.W.; Fisher, T.E.; Egbertson, M.S.; Payne, L.S.; Juare, Jr. J.P.; Vacca, J.P.; Hazuda, D.J.; Felock, P.J.; Wolfe, A.L.; Stillmock, K.A.; Witmer, M.V.; Moyer, G.; Schleif, W.A.; Gabryelsk, L.J.; Leonard, Y.M.; Lynch, Jr. J.J.; Michelson, S.R.; Young, S.D. Design and Synthesis of 8-Hydroxy-[1,6]Naphthyridines as Novel Inhibitors of HIV-1 Integrase in Vitro and in Infected Cells. J. Med. Chem. 2003, 46, 453-456.

16. Summa, V.; Petrocchi, A.; Matassa, V.G.; Taliani, M.; Laufer, R.; Francesco, R.D.; Altamnra, S.; Pace, P. HCV NS5b RNA-Dependent RNA Polymerase Inhibitors: From $\alpha, \gamma$-Diketoacids to 4,5Dihydroxypyrimidine- or 3-Methyl-5- hydroxypyrimidinonecarboxylic Acids. Design and Synthesis. J. Med. Chem. 2004, 47, 5336-5339.

17. Li, X.-M.; Zeng, C.-C.; Jiao, Z.-G.; Yan, H.; Zhong, R.-G. Synthesis and crystal structure of 7acetamido-5-chloro-8-(p-methyloxybenzenesulfonyloxy)-2-styrylquinoline. Chinese J. Struct. Chem. 2007, 26, 669-673.

18. Xu, Y.-S.; Zeng, C.-C.; Li, X.-M.; Zhong, R.-G.; Zeng, Y. Design, synthesis and Cu2+ recognition of $\beta$-diketoacid and quinoxalone derivatives bearing caffeoyl or galloyl moieties linked by arylamide as potential HIV integrase inhibitors. Chin. J. Chem. 2006, 24, 1086-1094.

19. Zeng, C.-C.; Li, X.-M.; Yan, H.; Zhong, R.-G. Design and synthesis of p/m-[p-(un)substituted phenylsulfonamido]phenyl $\beta$-diketo acids and quinoxalone derivatives. Chin. J. Chem. 2007, 25, 1174-1182.

20. Zeng, C.-C.; Liu, C.-F.; Zeng, J.; Zhong, R.-G. Electrochemical synthesis of 6-arylsulfonyl caffeic acid derivatives in aqueous medium. J. Electroanal. Chem. 2007, 608, 85-90.

21. Zeng, C.-C; Ping, D.-W.; Zhang, S.-C.; Zhong, R.-G.; Becker, J.Y. Electrochemical synthesis of polyhydroxylated aromatic derivatives substituted with mono- and dipyrimidinyl thioethers in aqueous medium. J. Electroanal. Chem. 2008, 622, 90-96.

22. Zeng, J.; Lv, X.-H.; Zeng, C.-C.; Hu. L.-M, Zhong, R.-G. Design, synthesis and anti-HIV integrase evaluation of 1,2,3-triazol -4-yl-substituted 1,4-dihydro-4-oxo-1,5-napthyridine-3carboxylic acids. Chin. J. Chem. 2009 (In press).

23. Komatsu, H.; Iwasawa, N.; Citterio, D.; Suzuki, Y.; Kubota, T.; Tokuno, K.; Kitamura, Y.; Oka, K.; Suzuki, K. Design and synthesis of highly sensitive and selective fluorescein-derived 
magnesium fluorescent probes and application to intracellular $3 \mathrm{D}_{\mathrm{Mg}^{2+}}$ imaging. J. Am. Chem. Soc. 2004, 126, 16353-16360.

24. Otten, P.A.; London, R.E.; Levy, L.A. 4-Oxo-4H-quinolizine-3-carboxylic acids as $\mathrm{Mg}^{2+}$ selective, fluorescent indicators. Bioconjugate Chem. 2001, 12, 203-212.

25. Li, Q.; Chu, D.T.W.; Claiborne, A.; Cooper, C.S.; Lee, C.M.; Raye, K.; Berst, K.B.; Donner, P.; Wang, W.; Hasvold, L.; Fung, A.; Ma, Z.; Tufano, M.; Flamma, R.; Shen, L.L.; Baranowski, J.; Nilius, A.; Alder, J.; Meulbroek, J.; Marsh, K.; Crowell, D.; Hui, Y.; Seif, L.; Melcher, L.M.; Henry, R.; Spanton, S.; Faghih, R.; Klein, L.L.; Ken Tanaka, S, Plattner, J.J. Synthesis and Structure-Activity Relationships of 2-Pyridones: A Novel Series of Potent DNA Gyrase Inhibitors as Antibacterial Agents. J. Med. Chem. 1996, 39, 3070-3088.

26. Oya, S.; Masuda, N.; Kuroki, Y.; Inoue, T.; Okudo, M.; Iwata, T.; Kokubo, K.; Mizuno, H.; Hagiwara, M. Preparation of 4-oxo-4H-quinolizine-3-carboxylic acid derivatives as antibacterial agents. Jpn. Kokai Tokkyo Koho 2003, JP 2003261566 A 20030919

27. Fukumoto, R.; Niwano, Y.; Kusakabe, H.; Chu, C.; Kimura, H.; Nagasawa, K.; Yanagihara, S.; Hirosawa, C.; Ishiduka, S. Preparation of 4-oxoquinolizines as antimicrobial agents. PCT Int. Appl. 2003, WO 2003029253

28. Iwamoto, K.; Araki, K.; Fujishima, H.; Shinkai, S. Fluorogenic calix[4]arene. J. Chem. Soc. Perkin Trans. 1. 1992, 1885-1887.

29. Kim, Y.H.; Youk, J.S.; Moon, S.Y.; Choe, J.I.; Chang, S.K. $\mathrm{Hg}^{2+}$-selective fluorogenic chemosensor derived from 8-Aminoquinoline. Chem. Lett. 2004, 33, 702-703.

30. Kim, S.J.; Kool, E.T. Sensing Metal Ions with DNA Building Blocks: Fluorescent Pyridobenzimidazole Nucleosides. J. Am. Chem. Soc. 2006, 128, 6164-6171.

31. Weatherhead-Kloster, R.A.; Selby, H.D.; Miller III, W.B.; Mash, E.A. Organic Crystal Engineering with 1,4-Piperazine-2,5-diones. 6. Studies of the Hydrogen-Bond Association of Cyclo[(2-methylamino-4,7-dimethoxyindan-2-carboxylicacid)(2-amino-4,7-dimethoxyindan-2carboxylic acid)]. J. Org. Chem. 2005, 70, 8693-8702.

32. Kawasuji, T.; Fuji, M.; Yoshinaga, T.; Sato, A.; Fujiwara, T.; Kiyama, R. A platform for designing HIV integrase inhibitors. A two-metal binding model as a potential mechanism of HIV integrase inhibitors. Bio. \& Med. Chem., 2006, 14, 8420-8429

Sample Availability: Samples of compounds 6-14 are available from the authors.

(C) 2009 by the authors; licensee Molecular Diversity Preservation International, Basel, Switzerland. This article is an open-access article distributed under the terms and conditions of the Creative Commons Attribution license (http://creativecommons.org/licenses/by/3.0/). 